\title{
Acupuncture for General Anxiety Disorders: A Meta-Analysis
}

\author{
Yujie $\mathrm{Li}^{1}$, Yarong Peng', Xu Dü,** \\ ${ }^{1}$ Shaanxi University of Chinese Medicine, Xianyang 712046, China. \\ ${ }^{2}$ The Second Affiliated Hospital of Shaanxi University of Traditional Chinese Medicine, Xianyang 712046, China.
}

How to cite this paper: Yujie Li, Yarong Peng, Xu Du. (2021) Acupuncture for General Anxiety Disorders: A Meta-Analysis. International Journal of Clinical and Experimental Medicine Research, 5(3), 335-343. DOI: $10.26855 / \mathrm{ijcemr} .2021 .07 .016$

Received: May 13, 2021

Accepted: June 8, 2021

Published: June 25, 2021

*Corresponding author: $\mathrm{Xu} \mathrm{Du}$, The Second Affiliated Hospital of Shaanxi University of Traditional Chinese Medicine, Xianyang 712046, China.

Email: dxdmzhnsh@sina.com

\begin{abstract}
Objective: To systematically evaluate the clinical efficacy of acupuncture for general anxiety disorders by using evidence-based medicine. Methods: Online databases such as CNKI, VIP, Wanfang and PubMed were searched by computer, and references of randomized controlled trials (RCTS) of acupuncture therapy for generalized anxiety disorder (GAD) were screened out. The retrieval time was from the establishment of the database to December 2020. The RevMan 5.3 software was used for Meta analysis of Hamilton Anxiety scale (HAMA), Self-rating Anxiety Scale (SAS), total clinical effectiveness and adverse reactions in included randomized controlled trial (RCTS) literature. Results: A total of 19 RCTS were included in this meta-analysis, involving 1,493 patients, including 772 in the acupuncture group and 721 in the western medicine group. The Meta-analysis results showed that the effective rate of acupuncture treatment of GAD patients was better than that of western medicine group [RR=1.06, 95\%CI $(1.02,1.10), \mathrm{P}=0.003$ ], and the adverse reactions were significantly lower than that of western medicine group [RR=0.25, 95\%CI $(0.11,0.53), \mathrm{P}=0.0004]$. However, there was no significant difference in HAMA [MD=-0.71, 95\%CI $(-2.25,0.83), \mathrm{P}=0.37]$ and SAS $[\mathrm{MD}=-0.64,95 \% \mathrm{Cl}(-2.52,1.24), \mathrm{P}=0.51]$ of $\mathrm{GAD}$ patients after treatment in outcome indicators. Conclusion: Acupuncture is better than western medicine in treating generalized anxiety disorder. However, the evidence of efficacy is limited and more high-quality, big-data, multicenter RCT literature is needed for further study.
\end{abstract}

\section{Keywords}

Acupuncture, General Anxiety Disorders, Clinical Effects, Meta Analysis

\section{Introduction}

General Anxiety disorder (GAD) is a kind of neurosis characterized by frequent or persistent excessive anxiety, worry, restlessness, and even fear with no definite object or fixed content. It is often accompanied by symptoms such as chest distress, palpitations, insomnia, hand shaking, muscle tension, dyspnea, and restlessness. Patients often feel painful because they are psychologically difficult to bear but cannot get rid of it, which affects their normal work and life. With the rapid development of modern society and the increasingly fierce social competition, more and more people have different degrees of anxiety. Domestic studies have shown that the lifetime prevalence of GAD is $4.1 \%$ to $6.6 \%$, the annual prevalence of general population is $1.9 \%$ to $5.1 \%$, and the prevalence rate of male and female is about 1:2 [1]. In particular, the recent outbreak of COVID-19,a public health emergency, has caused 
psychological and physical harm to people. Relevant studies [29] show that the incidence of anxiety disorders during the epidemic is $19.2 \%$, and the isolation time is positively correlated with the occurrence of anxiety disorders, especially in areas with severe epidemic. At present, the treatment of GAD mainly adopts anti-anxiety and depression drugs and psychotherapy. Anti-anxiety drugs have the advantages of quick effect, and studies have shown that the remission rate of drug therapy is $44 \%$ to $81 \%$ [2]. But this kind of drug has strong dependence and drug resistance. Still can cause the side effect such as the gitract reaction of different degree, memory to drop. Causes the patient's condition to recur even further aggravation. However, there is great uncertainty in the therapeutic effect of psychotherapy and it is difficult to measure the effect.

Therefore, finding the best treatment for GAD is an urgent problem. In recent years, acupuncture treatment of GAD in Chinese medicine has achieved good efficacy, but there is still a lack of definite evidence-based medicine evidence. Therefore, the author collected, extracted, systematically analyzed and evaluated the clinical efficacy of randomized controlled studies of acupuncture treatment GAD, in order to provide stronger and more scientific evidence-based medical evidence for the clinic.

\section{Data and methods}

\subsection{Source of data}

Online databases such as CNKI, VIP, Wanfang and PubMed were used to retrieve relevant literature materials.

\subsection{Retrieval strategies}

The subject term and free word were combined in retrieval. Searching terms included "acupuncture”, "anxiety” and "randomized controlled trial".

\subsection{Inclusion and exclusion criteria}

\subsubsection{Inclusion criteria}

(1) Type of research: RCT. whether blind or not;

(2) Observation object: gender, age, unlimited source of cases, according to the Chinese classification and diagnostic criteria for mental disorders version 3 (CCMD-III) diagnostic criteria [3], has been clear diagnosis of patients with GAD;

(3) Intervention measures: Acupuncture was used in the treatment group, and combined electroacupuncture was excluded. The control group was western medicine;

(4) Outcome indicators: Hamilton Anxiety Scale (HAMA), anxiety self-rating Scale (SAS), clinical efficiency and adverse reactions.

\subsubsection{Exclusion criteria}

(1) Literature for non-randomized controlled trials;

(2) The intervention measures were non-acupuncture literature, or the original literature research design is not rigorous, improper statistical methods or repeated published literature;

(3) Conference papers, dissertations, abstracts, etc;

(4) Animal experiment;

(5) Missing major outcome measures in the literature;

(6) The full text of the literature is not available;

(7) There is no clear diagnostic criterion in the literature.

\subsection{Data extraction and quality evaluation}

Noteexpress software is used to search the related literature rechecking, classification, will be included in the standard literature by two researchers using Cochrane recommended the Jadad scale for quality evaluation, in case of disagreement, the analysis of the two sides to discuss to come to an agreement or by a third, researchers to assist decision Jadad altogether four, scoring total score of 7 points, including 4 to 7 is divided into high quality documents, 1 to 3 are divided into low quality documents.

\subsection{Statistical approach}

In terms of data processing, RevMan5.3 software was used for Meta analysis. Enumeration data were represented by odds ratio (OR) OR relative risk (RR), measurement data by weighted mean difference (WMD) OR 
mean difference (SMD), and 95\% confidence interval (CI). Heterogeneity test was conducted by chi-squared test. When multiple studies of the same kind had homogeneity ( $P>0.05, \mathrm{I} 2 \leq 50 \%$ ), the combined statistics were determined by the fixed-effect model. On the contrary, random effects model is used. Funnel plot was used to analyze publication bias. A subgroup analysis was performed according to the factors that might lead to heterogeneity, and sensitivity analysis was performed when heterogeneity was derived from low-quality studies.

\section{Results}

\subsection{Literature search results}

A total of 459 articles related to Chinese and English were retrieved by computer and manual retrieval, including 168 articles on CNKI, 158 articles on Wanfang data, 70 articles on VIP and 63 articles on Pubmed. After reading the title, abstract and full text of the research literature, screening according to the research purpose, inclusion criteria and exclusion criteria, 19 RCT literatures were finally decided to be included [4-22]. The specific retrieval process is shown in Figure 1.

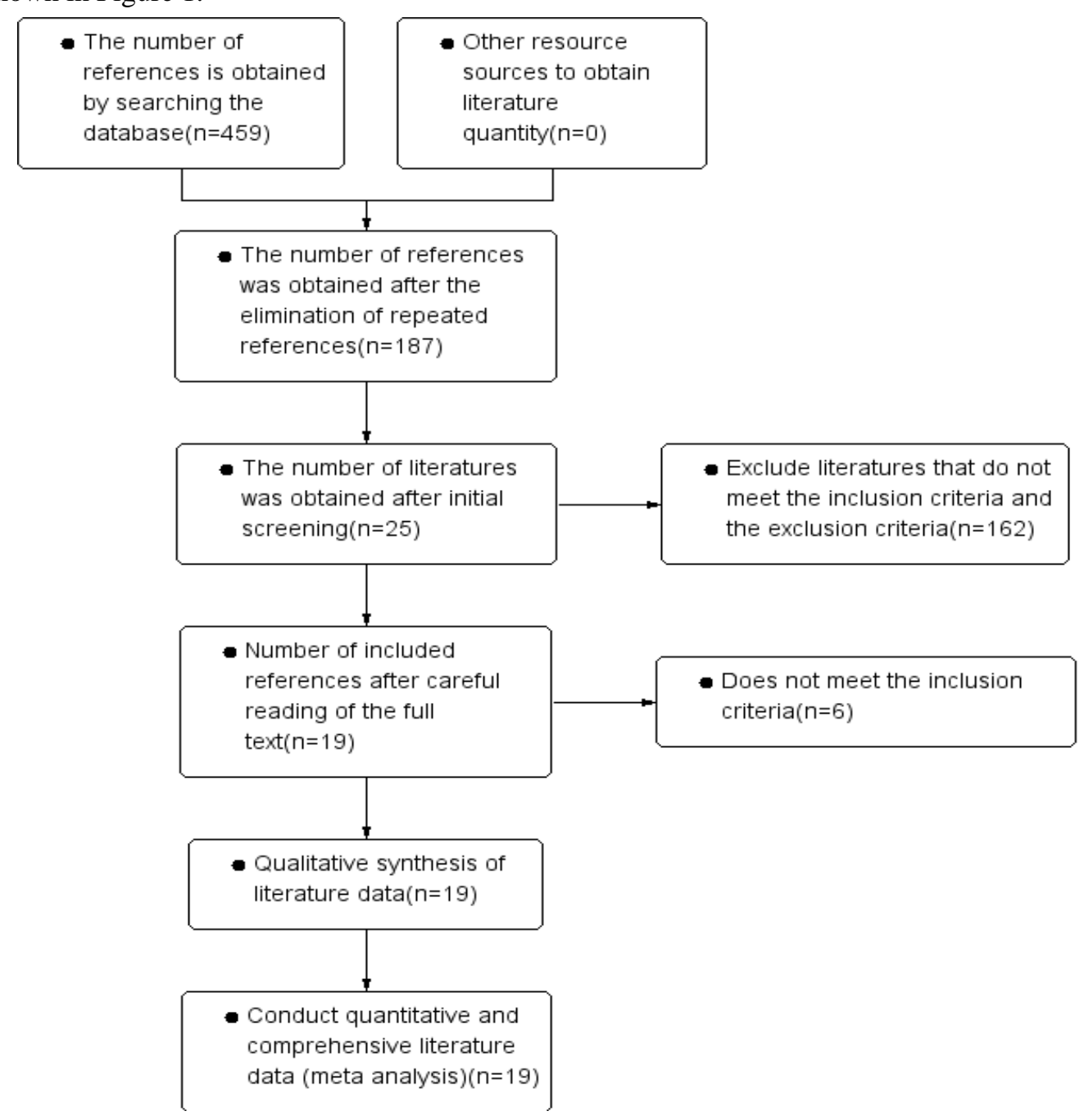

Figure 1. Literature screening flow chart.

\subsection{The basic characteristics of all included literatures}

In the 19 articles [4-22] included, a total of 1,493 patients were included, including 772 patients in the acupuncture group and 721 patients in the Western medicine group. Among them, 14 literatures [4-9, 11-12, 14-16, 21-22] reported the control of Hamilton Anxiety Scale (HAMA) before and after treatment,9 literatures [6-7, 10-13, 15-16, 21] reported the changes of anxiety Self-rating Scale (SAS), and 16 literatures [4-7, 9-16, 18-20, 22] reported the effective rate of treatment. See Table 1 for details and the results of the risk of bias assessment in Figure 2 . 
Table 1. Basic information of included literatures

\begin{tabular}{|c|c|c|c|c|c|c|}
\hline Authors & Country & $\begin{array}{c}\text { Diagnostic } \\
\text { Patients (T/C) }\end{array}$ & Duration & Acupuncture group & Western medicine group & $\begin{array}{l}\text { Outcome mea- } \\
\text { surements }\end{array}$ \\
\hline Li et al. 2013 [4] & China & $15 / 15$ & 8 weeks & $\begin{array}{l}\text { Acupuncture, ear point } \\
\text { tapping, Deanxit }\end{array}$ & Deanxit & (1) \\
\hline Luo et al. 2007 [5] & China & $29 / 29$ & 6 weeks & acupuncture & $\begin{array}{l}\text { Fluoxetine or paroxetine } \\
\text { and alprazolam }\end{array}$ & (1) \\
\hline Sui et al. 2010 [6] & China & $38 / 38$ & 6 weeks & acupuncture, paroxetine & paroxetine & (1) (3) \\
\hline Zhao et al. 2014 [7] & China & $30 / 30$ & 6 weeks & acupuncture & paroxetine & (1) (3) \\
\hline Ma et al. 2016 [8] & China & $30 / 30$ & 10 day & $\begin{array}{c}\text { acupuncture, manipulation, } \\
\text { paroxetine }\end{array}$ & paroxetine & (1) \\
\hline Wang et al. 2010 [9] & China & $30 / 30$ & 3 weeks & $\begin{array}{c}\text { acupuncture, } \\
\text { scraping therapy }\end{array}$ & alprazolam & (1) \\
\hline Zheng et al. 2014 [10] & China & $30 / 30$ & 4 weeks & acupuncture & ear point tapping & (2)(3) \\
\hline Zhang et al. 2010 [11] & China & $186 / 139$ & 30 days & $\begin{array}{c}\text { acupuncture, } \\
\text { acupoint injection }\end{array}$ & buspirone & (1) (3) \\
\hline Zheng et al. 2013 [12] & China & $30 / 30$ & 6 weeks & acupuncture, music & paroxetine & (1) (3) \\
\hline Xiong et al. 2013 [13] & China & $36 / 35$ & 60days & acupuncture & Deanxit & (2) \\
\hline Wang et al. 2007 [14] & China & $21 / 20$ & 30 days & acupuncture & Deanxit & (1) \\
\hline Liu et al. 2007 [15] & China & $29 / 29$ & 6 weeks & acupuncture & Fluoxetine or paroxetine & (1) (3) \\
\hline Che et al. 2015 [16] & China & $40 / 40$ & 6 weeks & acupuncture & paroxetine & (1) (3) \\
\hline Zhou et al. 2013 [17] & China & $40 / 40$ & 6 weeks & acupuncture & Clonazepam & (1) \\
\hline Gao et al. 2006 [18] & China & $42 / 45$ & 60 days & acupuncture, moxibustion & alprazolam & (2) \\
\hline Sun et al. 2015 [19] & China & $30 / 30$ & 4 weeks & acupuncture & Spirosterone citrate tablets & (3) \\
\hline Zhao et al. 2012 [20] & China & $45 / 40$ & 30 days & acupuncture & Deanxit & (4) \\
\hline Zhao et al. 2016 [21] & China & $41 / 41$ & 30 days & acupuncture & Deanxit & (1) \\
\hline Xu et al. 2016 [22] & China & $30 / 30$ & 30 days & $\begin{array}{l}\text { acupuncture, buspirone } \\
\text { hydrochloride tablets }\end{array}$ & $\begin{array}{l}\text { buspirone hydrochloride } \\
\text { tablets }\end{array}$ & (1) \\
\hline
\end{tabular}

Notes: (1). Hamilton Anxiety Scale (HAMA); (2). self-rating anxiety scale (SAS); (3). Efficient.

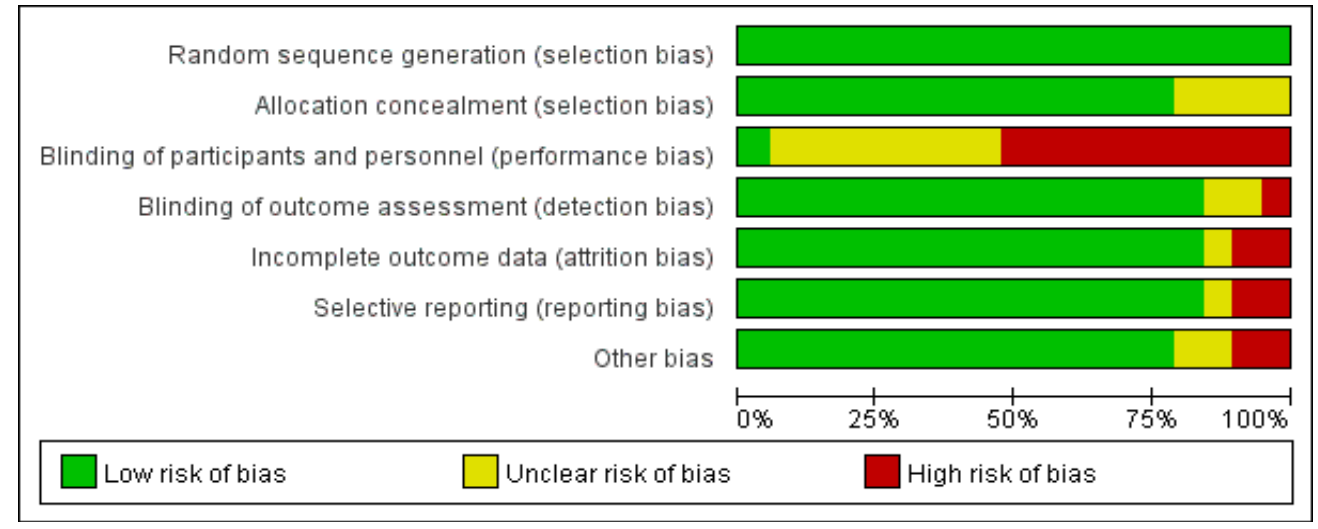

Figure 2. Risk of bias assessment of the included articles.

\subsection{Quality evaluation of included literature methodology}

After evaluating the methodological quality of the 19 references [4-22] included, it was found that there is a high quality [14] and the rest were of low quality. All the literatures are RCTS and mention the use of random methods for grouping, but the random methods are not described in detail. One article uses allocation hiding [15]. Three reports shedding/dropping [5, 15, 21]. Six articles mentioned adverse reactions [4, 7, 11, 15-16, 19], and one article [19] did not describe the number of adverse reactions in detail. See Table 2 for the specific situation of method 
ologicalquality evaluation in the included research literature.

Table 2. Methodological quality evaluation of the included studies

\begin{tabular}{|c|c|c|c|c|c|c|c|c|}
\hline Authors & random method & Baseline & $\begin{array}{c}\text { fol- } \\
\text { low-up }\end{array}$ & $\begin{array}{l}\text { adverse } \\
\text { reaction }\end{array}$ & $\begin{array}{c}\text { Fall } \\
\text { off/quit }\end{array}$ & $\begin{array}{l}\text { allocation } \\
\text { concealment }\end{array}$ & $\begin{array}{l}\text { blind } \\
\text { method }\end{array}$ & $\begin{array}{l}\text { The jadad } \\
\text { score }\end{array}$ \\
\hline Li et al. 2013 [4] & random & comparable & NR & YES & NR & NR & NR & 1 \\
\hline Luo et al. 2007 [5] & Random order of visits & comparable & NR & NR & YES & NR & NR & 3 \\
\hline Sui et al. 2010 [6] & random & comparable & NR & NR & NR & NR & NR & 1 \\
\hline Zhao et al. 2014 [7] & random number table & comparable & NR & YES & NR & NR & NR & 2 \\
\hline Ma et al. 2016 [8] & random & comparable & NR & NO & NR & NR & NR & 1 \\
\hline Wang et al. 2010 [9] & random & comparable & NR & NR & NR & NR & NR & 1 \\
\hline Zheng et al. 2014 [10] & random & comparable & NR & NR & NR & NR & NR & 1 \\
\hline Zhang et al. 2010 [11] & random number table & comparable & NR & YES & NR & NR & NR & 2 \\
\hline Zheng et al. 2013 [12] & random number table & comparable & NR & NR & NR & NR & NR & 2 \\
\hline Xiong et al. 2013 [13] & random & NR & NR & NR & NR & NR & NR & 1 \\
\hline Wang et al. 2007 [14] & Random order of visits & NR & NR & NR & NR & NR & NR & 2 \\
\hline Liu et al. 2007 [15] & Random seal card & comparable & NR & YES & YES & YES & YES & 5 \\
\hline Che et al. 2015 [16] & random & comparable & NR & YES & NR & NR & NR & 1 \\
\hline Zhou et al. 2013 [17] & Admission sequence & comparable & NR & NR & NR & NR & NR & 2 \\
\hline Gao et al. 2006 [18] & random & comparable & NO & NR & NR & NR & NR & 1 \\
\hline Sun et al. 2015 [19] & random number table & comparable & NO & YES & NR & NR & NR & 2 \\
\hline Zhao et al. 2012 [20] & random number table & NR & NO & NR & NR & NR & NR & 2 \\
\hline Zhao et al. 2016 [21] & random number table & comparable & NO & NR & YES & NR & NR & 3 \\
\hline Xu et al. 2016 [22] & random number table & comparable & NO & NR & NR & NR & NR & 2 \\
\hline
\end{tabular}

\subsection{Meta analysis results}

\subsubsection{Meta Analysis of Hamilton Anxiety Scale (HAMA)}

Fourteen articles [4-9, 11-12, 14-17, 21-22] included, a total of 1122 GAD patients, 586 patients in acupuncture group and 536 patients in western medicine group were included. Heterogeneity $\left(\mathrm{I}^{2}=91 \%, \mathrm{P}<0.00001\right)$ was observed in the 14 control groups. Random-effect model was used for Meta analysis. The results showed that there was no statistically significant difference between the two groups [MD=-0.71, 95\% CI(-2.25, 0.83), P =0.37], indicating that HAMA level in the acupuncture group and the Western medicine group in this study showed no statistical significance, that is, no significant difference in HAMA indication in treatment of GAD between acupuncture and western medicine (see Figure 3).

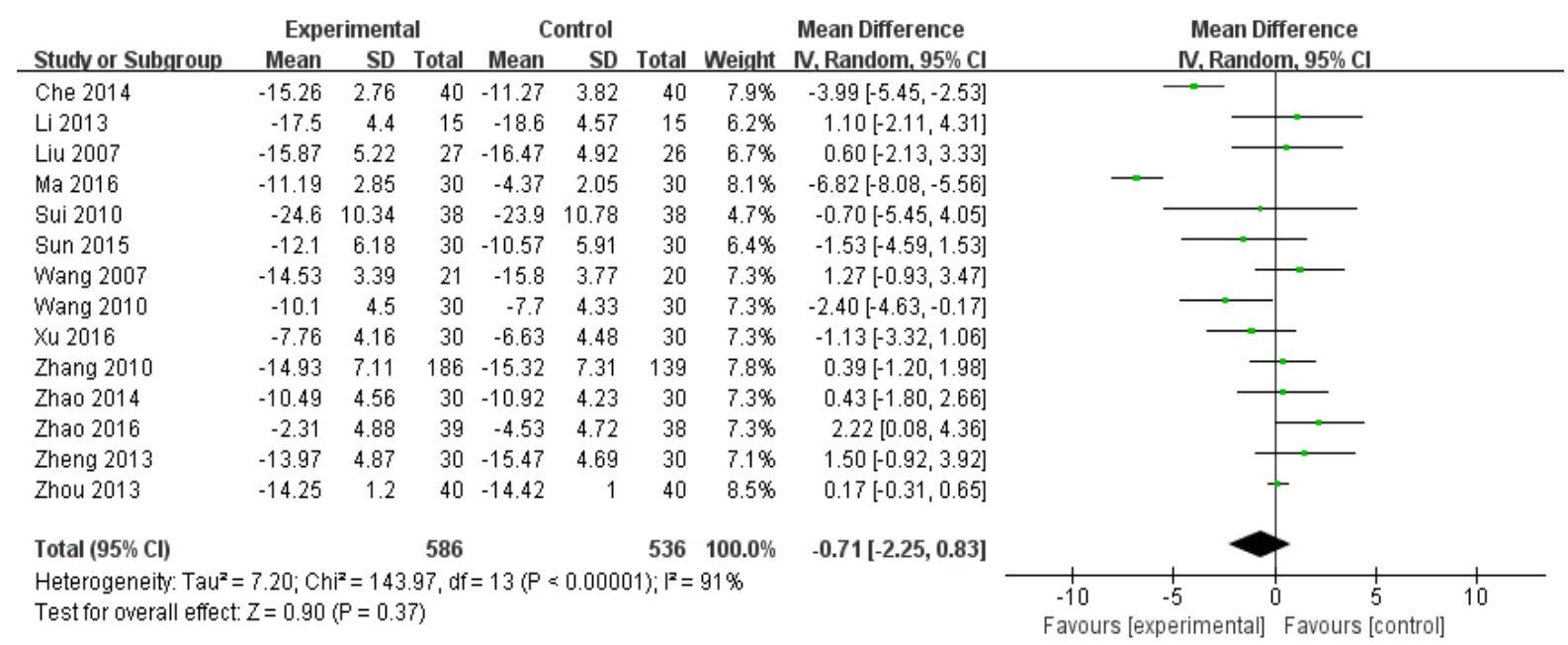

Figure 3. Forest map of HAMA level was compared between acupuncture group and Western medicine group. 


\subsubsection{Meta analysis of Self-rating Anxiety Scale (SAS)}

Nine articles included [6-7, 10-13, 15-16, 21], there were a total of 862 GAD patients, 456 patients in the acupuncture group, and 406 patients in the western medicine group. Heterogeneity was observed among the nine control groups $\left(\mathrm{I}^{2}=69 \%, \mathrm{P}=0.0010\right)$, so the random-effect model was used in the meta-analysis. The analysis results showed that there was no statistically significant difference between the two groups [MD=-0.64, 95\% Cl $(-2.52$, 1.24), $\mathrm{P}=0.51$ ], indicating that there was no statistically significant difference in SAS level between the acupuncture group and the western medicine group in this study $(\mathrm{P}>0.05)$ (see Figure 4$)$.

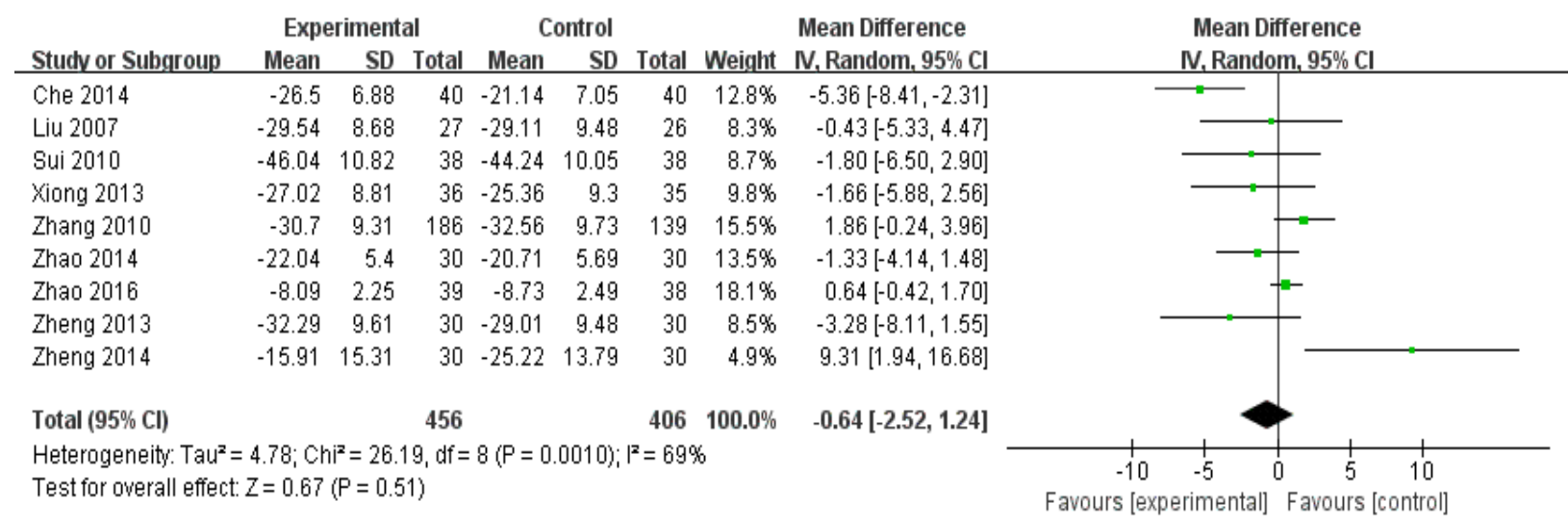

Figure 4. Forest map of acupuncture group and Western medicine group were compared in SAS level.

\subsubsection{Effective meta-analysis}

Sixteen articles included [4-7, 9-16, 18-20, 22], there were a total of 1213 GAD patients, 632 patients in the acupuncture group, and 581 patients in the western medicine group. There was no heterogeneity $\left(\mathrm{I}^{2}=5 \%, \mathrm{P}=0.39\right)$ in the 16 control groups, so fixed effect model was used in the meta-analysis. The analysis results showed that the difference between the two groups was statistically significant [RR=1.06, 95\% CI $(1.02,1.10), \mathrm{P}=0.003]$, indicating that acupuncture was significantly better than western medicine group in the effective treatment of GAD (see Figure 5).

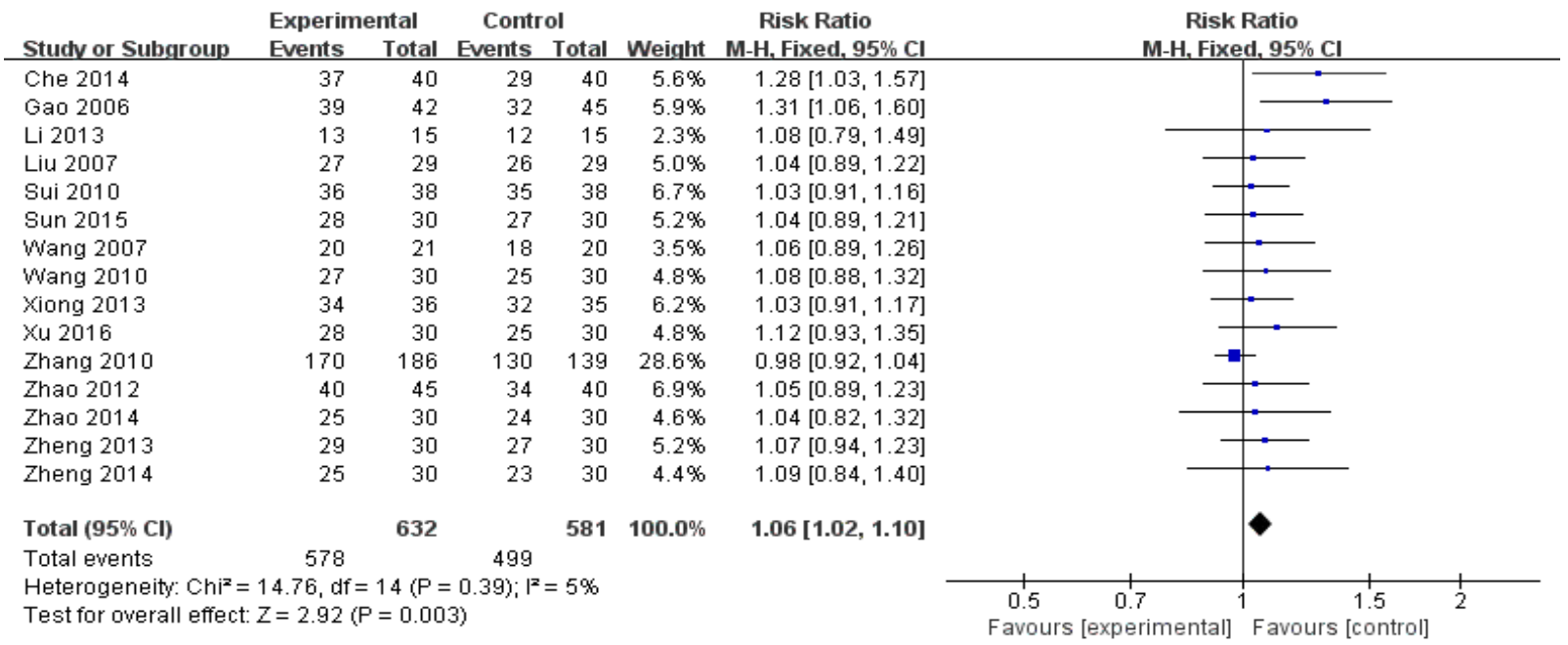

Figure 5. Forest map of comparison of effective rate between acupuncture group and Western medicine group.

\subsubsection{Meta-analysis of adverse reactions}

$5[4,7,11,15-16]$ of the included literatures reported the occurrence of adverse reactions in detail, including a total of 613 patients, including 330 in the acupuncture group and 283 in the Western medicine group. For heterogeneity test, $\mathrm{P}=0.02$ and $\mathrm{I}^{2}=62 \%$, suggesting that each study was heterogeneous, so the combined effect size was used by the random effect model $[\mathrm{RR}=0.25,95 \% \mathrm{CI}(0.11,0.53) \mathrm{P}=0.0004]$. The combined effect was located to the left of the invalid line of the forest map and did not intersect with the invalid line, suggesting that the adverse reaction of acupuncture group was significantly lower than that of western medicine group (see Figure 6). 


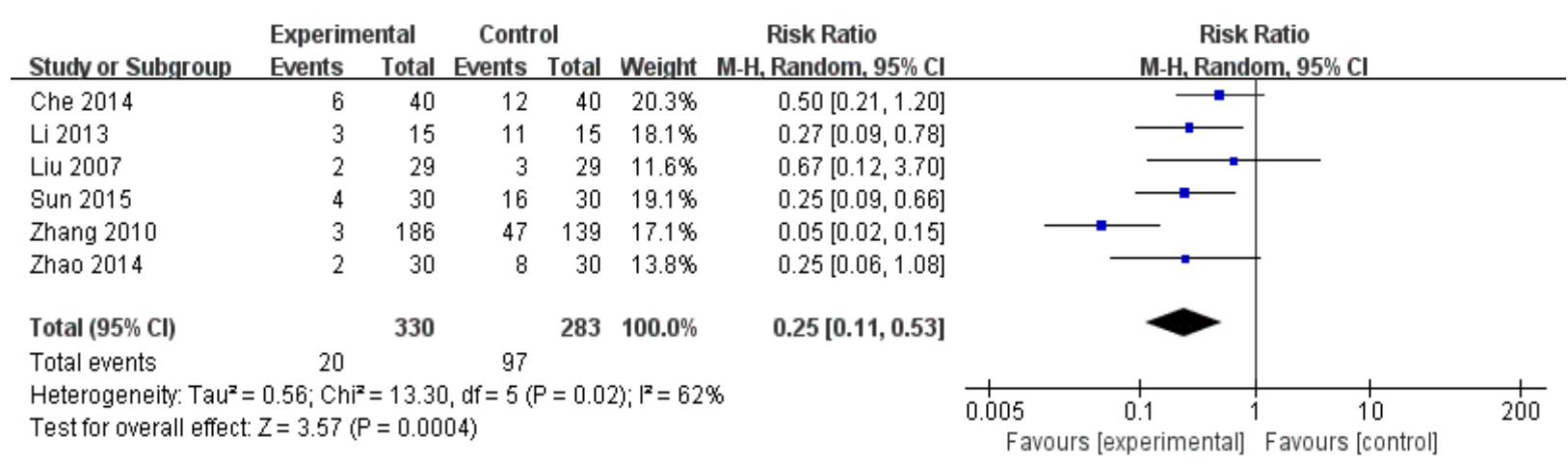

Figure 6. Forest map of adverse reactions between acupuncture group and Western medicine group.

\subsubsection{Adverse reaction symptoms}

$6[4,7,11,15-16,19]$ in the literature describing the adverse reactions of the two groups, the adverse reactions of the Western medicine group were mainly manifested as dizziness, lethargy, gastrointestinal reactions, dry mouth, fatigue, insomnia, headache, sweating, dyspnea, and one case withdrew from the study due to unbearable side effects. In the acupuncture group, only dizziness, mild hematoma, gastrointestinal reactions and exacerbation of one case were observed.

\subsubsection{Publication bias}

Funnel plots were drawn based on the results of total effective rate studies reported in 15 [4-7, 9-16, 18-20, 22]. The results suggested that the distribution of effective funnel plots was not symmetrical, indicating that there was a certain publication bias, which may be closely related to the low quality and small sample size of the literature included in this analysis (see Figure 7).

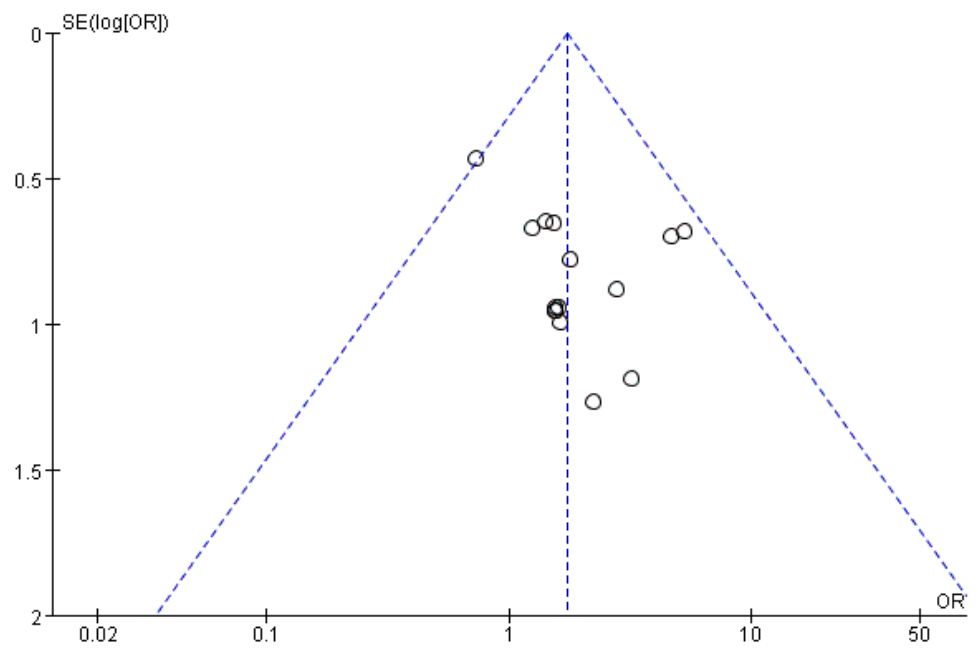

Figure 7. Funnel plot of the total clinical effective rate of GAD treated by acupuncture.

\subsubsection{Sensitivity analysis}

After the lower quality literature studies were excluded in turn, the combined effect size was re-estimated and compared with the previous meta-analysis results. The results showed that there was no significant change in the combined effect of clinical efficiency, indicating low sensitivity and the existence of bias had no substantial influence on the study results.

\section{Discussion}

GAD belongs to the category of emotion disease in Chinese medicine, which is equivalent to the depression syndrome and viscera disorder in Chinese medicine. According to the Theory of Yin and Yang should be like the Big Picture, it says: "People have five zang and five Qi, so as to produce emotions, emotions, worries and fears". So GAD associated with viscera, especially to close to the heart, liver, spleen, kidney, mostly due to the thought of modern shu, excessive, diseases with ease, seven emotions, heart disorder of primary causes, such as research [30] 
showed that the incidence of GAD female than for male, with the structure of human brain function, synthesis of serotonin in the body, and hormonal differences are closely related. Clinical to tune god, which is "all the true thorns, must first cure God" embodiment, and conditioning the zang-fu combined. Specifically, acupuncture points such as Bahui, Sishencong and Yintang to calm and calm the mind, Shenmen, Neiguan, Xinshu and other points to calm the mind and calm the mind, taichong to soothe the liver and relieve depression to alleviate the patient's symptoms. At the same time, can treat a series of symptoms such as insomnia, eating less na stay, chest tightness. Other studies have shown that outdoor exercise can improve patients' bad mood by releasing 5-hydroxytryptamine and endorphins in the body [23]. GAD patients should take appropriate outdoor activities, such as Baduanjin and Taiji sword, to distract their attention and eliminate bad emotions.

The results of this meta analysis showed that HAMA and SAS indicators could not clearly indicate the significant advantages of acupuncture compared with western medicine in the treatment of generalized anxiety disorder. However, the overall efficacy of acupuncture was better than that of western medicine group in terms of effective rate and occurrence of adverse reactions. In recent years, many clinical [24-26] and experimental studies [27-28] have further shown that acupuncture can not only improve patients' clinical symptoms, reduce the recurrence rate and improve the quality of life, but also avoid the adverse reactions brought by western medicine treatment, with good safety. Moreover, acupuncture has the advantages of simple operation and low cost, so acupuncture has a great advantage in treating GAD.

Deficiencies of this meta-analysis: (1) of the 19 articles included, only 1 was of high quality, and the methodological quality of the included studies was generally low. Although all the 19 articles described randomness, only one used the method of allocation concealment, and the rest did not describe the specific random method. Therefore, it is impossible to determine whether the included literature truly achieves randomness, and it is also impossible to rule out whether the researchers' selection bias is caused by objective or subjective factors. (2) The sample size of the included study was small, the study time was relatively short, and the lack of follow-up made it impossible to make a scientific and efficient evaluation of its long-term efficacy. (3) The included studies were all Chinese literature, which could not comprehensively analyze the efficacy of acupuncture on various populations. Therefore, in the future, multi-center, large sample and high-quality studies are needed to further provide stronger clinical evidence.

To sum up, acupuncture has a significant effect on the improvement of symptoms in GAD patients, and is one of the effective methods to treat GAD. However, multi-center, large sample size and high-quality literature are still needed to further confirm, so as to provide strong evidence-based medical evidence for clinical practice.

\section{References}

[1] Wu, W. Y. (2010). Guidelines on prevention and Treatment of Anxiety Disorders [M]. Beijing: People’s Medical Publishing House, 2010: 115.

[2] Bandelow Borwin, Boerner J. Reinhard, Kasper Siegfried, Linden Michael, Wittchen Hans-Ulrich, Möller Hans-Jürgen. (2013). The diagnosis and treatment of generalized anxiety disorder [J]. Deutsches Ärzteblatt International, 2013, 110(17).

[3] Chinese Psychiatric Association. (2001). Ccmd-3 Classification and Diagnostic Criteria of Mental Disorders in China [M]. 3rd Ed. Jinan: Shandong Science and Technology Press, 2001: 89-90.

[4] Li, L. F., Yang, Y. H., Yin, G. L. (2013). Clinical Observation on the Treatment of menopause Anxiety by ear pressure and acupuncture combined with Deli New therapy [J]. New Chinese Medicine, 2013, (5): 124-126.

[5] Luo, W. Z., Liu, H. J., Mei, S. Y., et al. (2007). Jin S S clinical observation on the treatment of generalized anxiety disorder [J]. Chinese Journal of Integrated Traditional and Western Medicine, 2007, (3): 201-203.

[6] Sui, A. M. (2010). Therapeutic effect of Paroxetine combined with acupuncture on 38 cases of anxiety [J]. Fujian Chinese Medicine, 2010, (3).

[7] Zhao, Y., Zou, W., Teng, W., et al. (2014). Clinical study on the Treatment of Generalized Anxiety Disorder with TongDu and Shen-Regulating Acupuncture [J]. Chinese Journal of Acupuncture and Moxibustion, 2014, (11): 24-26.

[8] Ma, Y., Liu, J. C., Liang, X. Y., et al. (2016). Chinese Journal of Traditional Chinese Medicine, 2016, (11): $4863-4865$.

[9] Wang, Y. N., Zheng, Z. Y. (2010). Research on the clinical effect of Acupuncture at Dorsal Shu Point for treating anxiety neurosis [J]. Chinese Journal of Acupuncture and Moxibustion, 2010, (9): 13-14.

[10] Zheng, Z. Y., Wang, S., Miao Yongxin. (2014). A comparative study of acupuncture at Back Shu point and auricular Point sticking pressure in the treatment of generalized Anxiety disorder [J]. Journal of Acupuncture and Moxibustion, 2014, (12): 33-34.

[11] Zhang, H., Deng, H. (2010). Effect observation of acupuncture combined with acupoint injection in the treatment of 
post-earthquake Generalized Anxiety disorder [J]. Shanghai Journal of Acupuncture and Moxibustion, 2010, (9): $576-577$.

[12] Zheng, W., Liu, H. J., Qi, J., et al. (2013). Therapeutic Effect of acupuncture combined with music Therapy on Generalized Anxiety disorder [J]. Shanghai Journal of Acupuncture and Moxibustion, 2013, (11): 897-898.

[13] Xiong, Y. Q. (2013). Clinical Observation of acupuncture and Moxibustion for the Treatment of Generalized Anxiety disorder [J]. Guangming Chinese Medicine, 2013, (12): 2589-2590.

[14] Wang, G. M. (2007). Acupuncture treatment of 21 cases of generalized anxiety disorder [J]. Henan Traditional Chinese Medicine, 2007, (7): 55-56.

[15] Liu, H. J., Luo, W. Z., Mei, S. Y., et al. (2015). Journal of Guangzhou University of Traditional Chinese Medicine, 2007, (2): 119-122.

[16] Che, L. N. (2015). Effect of acupuncture on patients with generalized anxiety disorder [J]. China Minkang Medical Science, 2015, (8): 86-87.

[17] Zhou, X. F., Li Yan, Zhu Hong, et al. (2013). Effects of acupuncture on brain waves of patients with generalized anxiety disorder [J]. Chinese Acupuncture, 2013, (5): 395-398.

[18] Gao, L. P., Zhou, Y. (2006). Department of Medicine, Taizhou College of Vocational Technology, Jiangsu 225300. Observations on the Efficacy of Mind-Calming and Brain-Refreshing Acupuncture and Moxibustion for Treating 42 Anxiety Patients [J]. Acupuncture and Massage Medicine, 2006, (5): 300-302.

[19] Sun, Y. Z., Song, W. A., Ding, N. (2015). Clinical observation of yuanluo Tongjing acupuncture in the treatment of post-stroke anxiety [J]. Chinese Journal of Acupuncture and Moxibustion, 2015, (5): 32-34.

[20] Zhao, W. H., Zheng, X. Y. (2012). Acupuncture treatment of anxiety and depression after stroke: a total of 45 cases [J]. Chinese Journal of Acupuncture and Moxibustion, 2012, (12): 7-8.

[21] Zhao, Y., Wang, S. (2016). Influence of acupuncture on anxiety and depression caused by vertebrobasilar artery insufficiency vertigo [J]. Shanghai Journal of Acupuncture, 2016, (3): 282-283.

[22] Xu, M. M., Zhou, J. C., Liu, Y., et al. (2016). Observation on the efficacy of long-stay acupuncture combined with drugs in the emotional area of the head in the treatment of generalized anxiety disorder [J]. Shanghai Journal of Acupuncture and Moxibustion, 2016, (11): 1281-1283.

[23] Zhang, J., Zhang, W. W., Shen, H. (2016). Effect of practicing ba duan jin on the clinical efficacy of generalized anxiety disorder [J]. Chinese Journal of Sports Medicine, 2016, 35(03): 231-233.

[24] Xiao, X., Hu, C., Chen, K. X., et al. (2015). A Brief Analysis of the basis and progress of anti-anxiety acupuncture at Shenmenneiguan [J]. Yunnan Journal of Traditional Chinese Medicine, 2015, (11): 75-77.

[25] Zhao, L. G., Shi, R. X., Wu, Q., et al. (2011). Clinical Journal of Acupuncture and Moxibustion, 2011, (6): 35-36.

[26] Cao, T. J., Huang, F., Li, X., et al. (2007). Clinical Observation on the Treatment of depression from strange Classics [J]. Chinese Journal of Traditional Chinese Medicine, 2007, (7): 1401-1402.

[27] Xing, Y. S., Zheng, K. W., Liang, Q. C., et al. (2019). Therapeutic effect of acupuncture on alcohol withdrawal anxiety in rats and its relationship with CRH [J]. China Medical Journal, 2019, (26): 12-16.

[28] He, J. C. (2018). Effect of acupuncture on signaling molecules in PACAP/PAC1-PKA-CREB pathway in the nucleus of finstriated bed in anxious mice [D]. Chengdu University of Chinese Medicine, 2018.

[29] Li, Y. F., Wang, X. Y., Zhang, J., et al. (2020). Psychological survey of the general population during COVID-19 [J]. International Epidemiology: Electronic Edition, 2020, (2): 308-310.

[30] Cheng, G., Xiao, Y. Q. (2015). Gender differences in social anxiety disorders and their Biological explanations [J]. Chinese Journal of Health Psychology, 2015, (2): 307-312. 